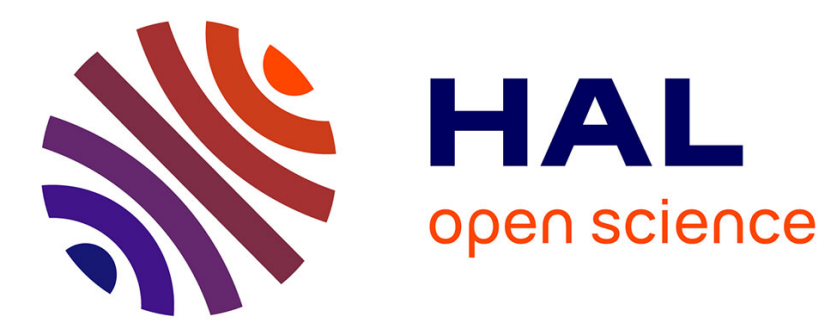

\title{
L'étude des tissus calcifiés par spectroscopie infrarouge photoacoustique
}

\author{
M. Di Renzo, T. Ellis, E. Sacher, I. Stangel
}

\section{To cite this version:}

M. Di Renzo, T. Ellis, E. Sacher, I. Stangel. L'étude des tissus calcifiés par spectroscopie infrarouge photoacoustique. Journal de Physique IV Proceedings, 1997, 07 (C6), pp.C6-221-C6-228. 10.1051/jp4:1997618 . jpa-00255717

\section{HAL Id: jpa-00255717 https://hal.science/jpa-00255717}

Submitted on 1 Jan 1997

HAL is a multi-disciplinary open access archive for the deposit and dissemination of scientific research documents, whether they are published or not. The documents may come from teaching and research institutions in France or abroad, or from public or private research centers.
L'archive ouverte pluridisciplinaire HAL, est destinée au dépôt et à la diffusion de documents scientifiques de niveau recherche, publiés ou non, émanant des établissements d'enseignement et de recherche français ou étrangers, des laboratoires publics ou privés. 


\title{
L'étude des tissus calcifiés par spectroscopie infrarouge photoacoustique
}

\author{
M. di Renzo*****, T.H. Ellis*****, E. Sacher****** et I. Stangel*** \\ * Département de Chimie, Faculté des Arts et des Sciencès, Université de Montréal, CP. 6128, \\ Succursale Centre-Ville, Montréal, Québec H3C 3J7, Canada \\ ** Département de Génie Physique, École Polytechnique, Montréal, Québec H3C 3A7, Canada \\ *** Faculty of Dentistry, McGill University, 740 av. Dr. Penfield, Montréal, Québec H3A 1A4, Canada \\ **** Groupe de Recherche en Physique et Technologie des Couches Minces (GCM)
}

\begin{abstract}
Résumé: Notre travail implique l'étude des modifications advenant à la dentine humaine lors de son exposition à différents produits dans le but ultime de développer une meilleure adhérence à ce substrat lors de l'application de restaurations. Une meilleure adhérence entre la dentine et la restauration résultera en une augmentation de son étanchéité et donc de sa durabilité. Nous avons choisi la spectroscopie infrarouge photoacoustique (PA-FTIR) afin d'étudier les modifications apportées à la dentine lorsque celle-ci est traitée par des solutions acidiques ou d'hypochlorite de sodium $(\mathrm{NaOCl})$. La PA-FTIR s'avère une technique de choix pour l'étude des surfaces hétérogènes puisqu'elle ne demande aucune préparation ou modification de l'échantillon. Les spectres provenant des échantillons traités à l'acide démontrent une dissolution graduelle de la phase minérale de la dentine. Le traitement par $\mathrm{NaOCl}$ dissout le collagène et laisse derrière la phase minérale. Dans une dernière expérience, le traitement d'un disque de dentine à l'acide et à l'hypochlorite rétablit la surface de la dentine à son état non traité. Aucun changement n'a été remarqué pour des temps d'exposition plus longs (jusqu'à 15 minutes). Ce travail démontre l'utilité de la spectroscopie infrarouge photoacoustique envers l'étude des modifications chimiques des surfaces et interfaces des tissus calcifiés.
\end{abstract}

\section{INTRODUCTION}

L'étude des tissus biologiques calcifiés (os, dentine) a une place importante dans le développement de procédures permettant la réparation de ces tissus. Une meilleure compréhension de ces matériaux et des interactions entre ceux-ci et différentes substances mène au développement de nouvelles techniques et produits visant à réparer ou restaurer les tissus endommagés. En médecine dentaire, l'étude de la dentine a une place importante ayant comme but la création de restaurations étanches et durables. Malheureusement, quoique la morphologie de la dentine ait été bien caractérisée, sa chimie est encore mal comprise.

Une étape importante à la bonne adhérence des restaurations à la dentine humaine est un traitement à l'acide (mordançage). Cette étape consiste à traiter la surface avec un acide afin d'enlever la couche de débris de fraisage ainsi que déminéraliser la surface de la dentine. Cette couche de débris est crée lors du fraisage de la matière cariée de la dent et est généralement enlevée afin d'améliorer la rétention entre la dent et la restauration. De plus, les acides décalcifient la surface de la dentine et exposent le réseau organique composé principalement de collagène de type I $[1,2,3,4]$. Cette décalcification est proposée comme étant nécessaire à l'obtention d'une bonne interaction physicochimique entre le monomère adhésif et la dentine $[5,6]$. Avec la 
présente génération de systèmes adhésifs dentaires, le mordançage à l'acide est une étape primordiale à l'obtention de restaurations étanches et durables.

La majorité des travaux accomplis sur le mordançage portent sur la détermination de la concentration optimale d'acide ou sur les effets qu'a le mordançage sur la morphologie de la dentine $[1,2,3,4,7,8,9,10]$. Ces travaux touchent rarement aux effets chimiques produits sur la dentine au delà de quelques équations de dissolution ou de la cinétique. La dissolution de la phase minérale de la dentine suit une réaction contrôlée par diffusion $[10,11]$ et l'acidité est généralement le facteur le plus important lors de la dissolution de l'hydroxyapatite de calcium (HAP). Néanmoins, nous avons démontré que certains acides (citrique et phosphorique) forment des espèces insolubles au front de dissolution. Ces espèces peuvent gêner le processus de dissolution et ainsi ralentir le mordançage.

Ce travail tente de comprendre les modifications chimiques advenant à la dentine lorsqu'elle est traitée par différents acides à $\mathrm{pH}$ semblable. De plus, nous étudierons la possibilité d'enlever la phase complémentaire à HAP, soit le collagène, par l'utilisation de l'hypochlorite de sodium. Pour réaliser ce travail, nous avons choisi la spectroscopie infrarouge photoacoustique par transformée de Fourier (PA-FTIR), une technique sensible aux surfaces, afin de suivre les modifications de la dentine lors de différentes durées d'exposition aux acides ou à l'hypochlorite.

La technique de PA-FTIR a déjà été utilisée pour caractériser la composition chimique de la dentine $[6,12,13,14,15]$ et présente de nombreux avantages sur les autres techniques $[13,14]$. Parmi ceux-ci, on peut compter la possibilité d'obtenir des spectres de substrats opaques, tel que la dentine. Aussi, cette technique ne requiert aucune préparation de l'échantillon.

\section{PARTIE EXPÉRIMENTALE}

Des solutions acides (citrique, maléique et phosphorique) ont été préparées en diluant ou dissolvant l'acide dans l'eau purifiée par le processus Millipore. Le $\mathrm{pH}$ a été mesuré par un $\mathrm{pH}-$ mètre calibré muni d'une électrode de verre. Chaque acide a été ajusté a un $\mathrm{pH}$ de 1.0 par ajout d'eau. L'hypochlorite de sodium a été utilisé tel quel (eau de javel $12 \% \mathrm{p} / \mathrm{v}$ ).

Les échantillons étaient des disques de dentine provenant de dents humaines fraîchement extraites. Chaque disque à mordancer a été plongé dans une des solutions acides pendant un intervalle de temps totalisant $\mathrm{t}=0,10 \mathrm{~s}, 30 \mathrm{~s}, 1 \mathrm{~min}, 2 \mathrm{~min}, 3 \mathrm{~min}, 5 \mathrm{~min}, 11 \mathrm{~min}$ et $15 \mathrm{~min}$. À la fin de chaque temps d'exposition, l'échantillon a été rincé et sèché sous vide. Puisque les spectres initiaux du traitement à l'hypochlorite ont démontré un temps de réaction plus lent, l'échantillon traité avec ce produit a été exposé pendant des intervalles de $t=1,2$ heures, 1,2 et 6 jours. Dans une dernière expérience, un échantillon a été traité initialement à l'acide puis à l'hypochlorite (2 minutes chaque).

La technique de PA-FTIR se base sur la mesure d'une variation périodique de la pression d'un gaz situé au-dessus d'un échantillon (figure 1). Cette váriation de pression est causée par un dégagement de chaleur de l'échantillon lors de l'absorption de radiation infrarouge. L'énergie absorbée par l'échantillon diffuse vers la surface sous forme de chaleur, et chauffe une couche de gaz au-dessus de cette surface. Ce dégagement de chaleur crée des oscillations de température de 
la couche de gaz. Chacune de ces oscillations est associée à une longueur d'onde d'énergie absorbée.. Le signal photoacoustique, qui est détectée par un microphone, est généré par la somme de ces oscillations.

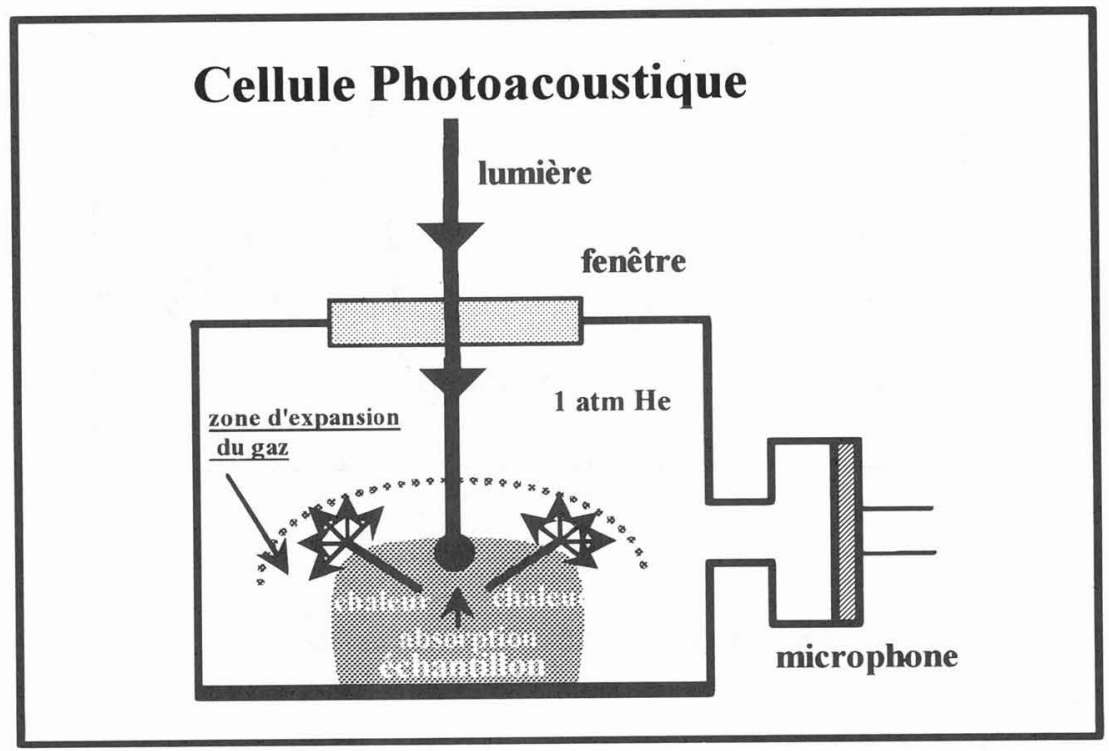

Figure 1: Schéma d'une cellule et du processus photoacoustique idéalisé.

Les échantillons ont été placés dans le porte-échantillon et insérés dans la cellule photoacoustique de modèle 300 de MTEC. Tous les échantillons ont été purgés à l'hélium avant de fermer la cellule. Les spectres ont été enregistrés à l'aide d'un spectrophotomètre FTIR Mattson RS-1 entre 500 et $4000 \mathrm{~cm}^{-1}$, à une vitesse de déplacement de miroir de $1.6 \mathrm{kHz}(0.098 \mathrm{~cm} / \mathrm{s})$ et une résolution de $8 \mathrm{~cm}^{-1}$. Cinq cents balayages ont été additionnés afin d'améliorer le rapport signal sur bruit. Tous les spectres ont été normalisé par rapport à la référence de noir de carbone fournie par MTEC.

\section{RÉSULTATS}

Les spectres du traitement de la dentine à l'acide maléique entre 0 et 1 minute sont présentés à la figure 2. On remarque une croissance de l'intensité du signal à travers le domaine spectral qui augmente avec le temps de traitement. Les spectres des temps de traitement plus longs indiquent encore une augmentation d'intensité qui n'est toutefois pas aussi prononcée qu'aux temps de traitement plus courts. De plus, il est possible de constater la diminution des pics à 1100 et 1445 $\mathrm{cm}^{-1}$. 


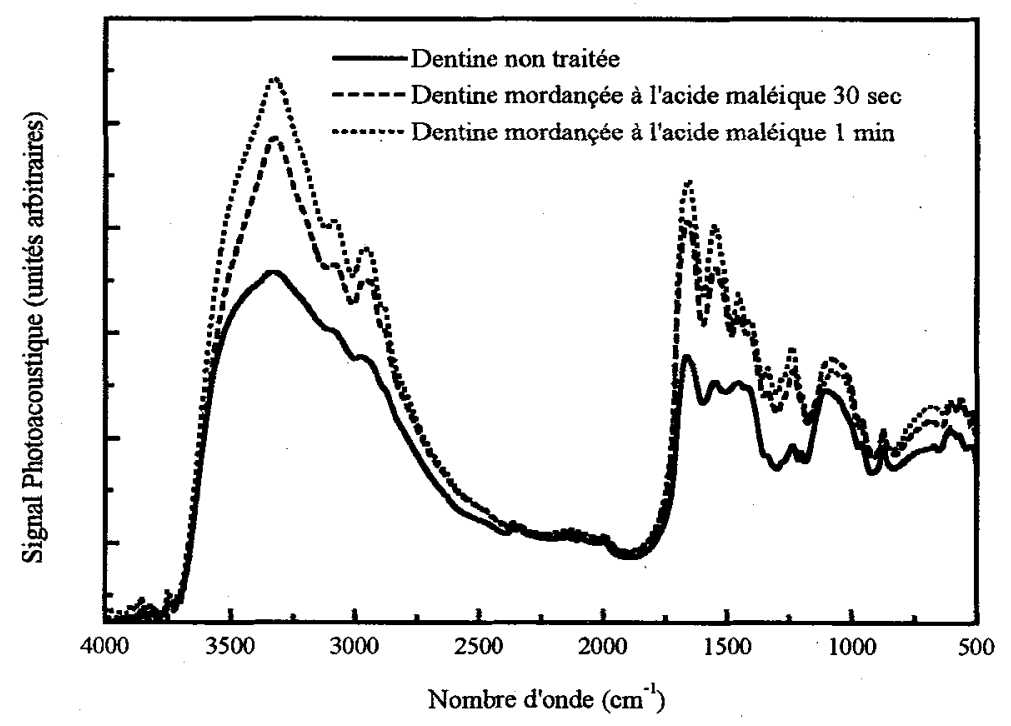

Figure 2: Spectres PA-FTIR de la dentine mordancée.

Afin de mieux observer ces changements, les spectres ont été normalisés à la bande amide I du collagène (ca. $1660 \mathrm{~cm}^{-1}$ ). Les résultats de cette normalisation pour les spectres de 0 à 1 minute se retrouvent à la figure 3 . Les pics situés à 1100 et $1445 \mathrm{~cm}^{-1}$ ont clairement diminué, particulièrement durant le traitement initial, soit les trente premières secondes. De plus, on peut constater la disparition de l'épaule située à $3550 \mathrm{~cm}^{-1}$ de la large bande autour de $3300 \mathrm{~cm}^{-1}$. Toutes ces bandes sont assignées à la phase minérale de la dentine. Le pic à $1100 \mathrm{~cm}^{-1}$ appartient à l'allongement du groupement phosphate de HAP. L'épaule à ca. $3550 \mathrm{~cm}^{-1}$ est assignée à l'allongement du groupe hydroxyle $(\mathrm{OH})$. La bande autour de $1445 \mathrm{~cm}^{-1}$ est le résultat de vibrations carbonate. L'existence d'apatites biologiques contenant du carbonate est bien documentée dans la littérature $[16,17,18,19,20]$. Des résultats semblables ont été trouvés pour l'acide phosphorique. Les spectres de l'acide citrique indiquent que, pour un temps de traitement identique, l'acide citrique a enlevé qualitativement moins de phase minérale.

Les résultats de la dentine traitée à l'hypochlorite de sodium sont présentés à la figure 4. Puisque HAP est insoluble à l'hypochlorite, la bande d'allongement du groupement phosphate $\left(1100 \mathrm{~cm}^{-1}\right)$ nous donne une intensité constante afin de normaliser les spectres. Ces spectres indiquent clairement l'enlèvement graduel de la matière organique par rapport au temps d'exposition (voir, par exemple, le pic amide I à $1660 \mathrm{~cm}^{-1}$ ). Les bandes de phosphate et de carbonate (1100 et 1445 $\mathrm{cm}^{-1}$ respectivement) deviennent plus apparentes avec le temps. 


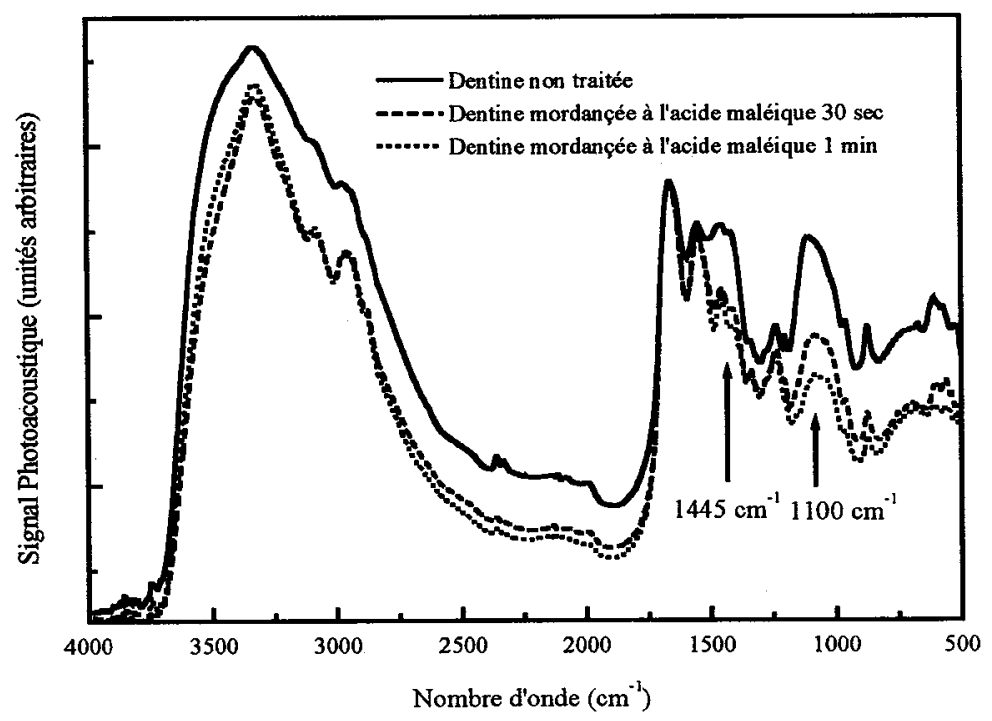

Figure 3: Spectres PA-FTIR normalisés de la dentine mordancée.

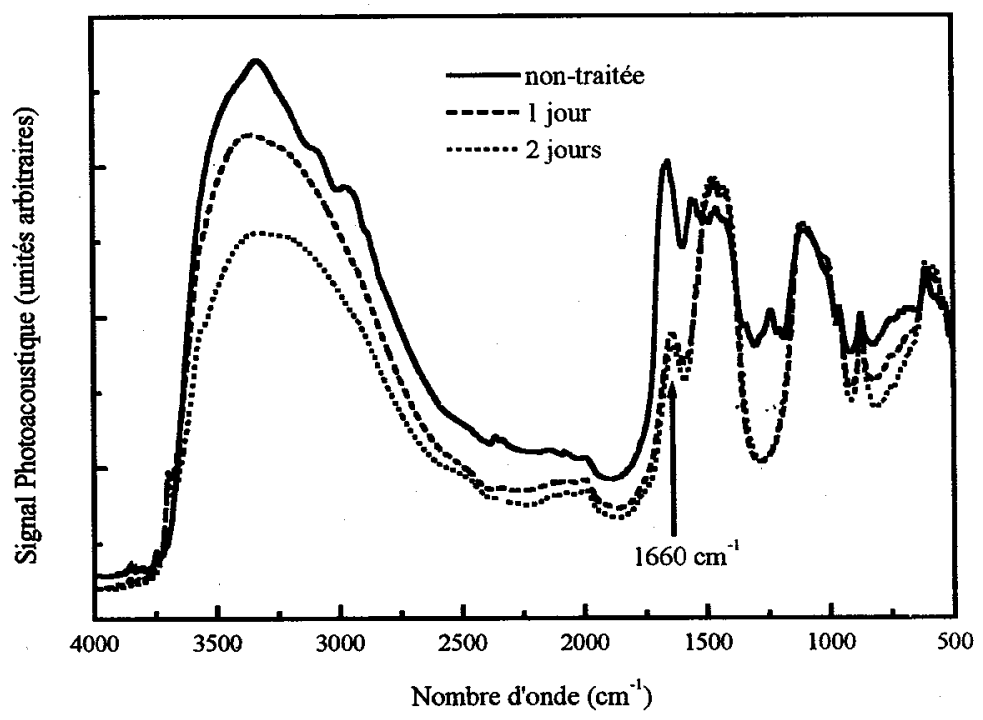

Figure 4: Spectres PA-FTIR normalisés de la dentine traitée à l'hypochlorite de sodium. 
Par contre, les vibrations de l'hydroxyle à $3550 \mathrm{~cm}^{-1}$ sont difficiles à repérer. Ce phénomène à déjà été observé chez les apatites préparés synthétiquement à basse température $[21,22,23]$. Les apatites biologiques ressemblent à ces apatites synthétiques qui ont une surface très élevée. Il est aussi possible de constater que le collagène est encore présent dans la région sondée, et ce, même après six jours.

Dans la dernière expérience, un échantillon de dentine a été mordancé à l'acide maléique pendant deux minutes $(\mathrm{pH}=1.0)$, rincé et sèché. Suite à ce traitement, un spectre de cet échantillon a été enregistré. Un spectre précédant tout traitement à aussi été obtenu. L'échantillon mordancé a ensuite été traité à l'hypochlorite pendant des périodes totalisant 0,10 et 30 secondes, $1,2,5$ et 15 minutes. À chaque intervalle, l'échantillon a été rincé et sèché avant d'ènregistrer son spectre. Certains de ces spectres sont présentés à la figure 5 .

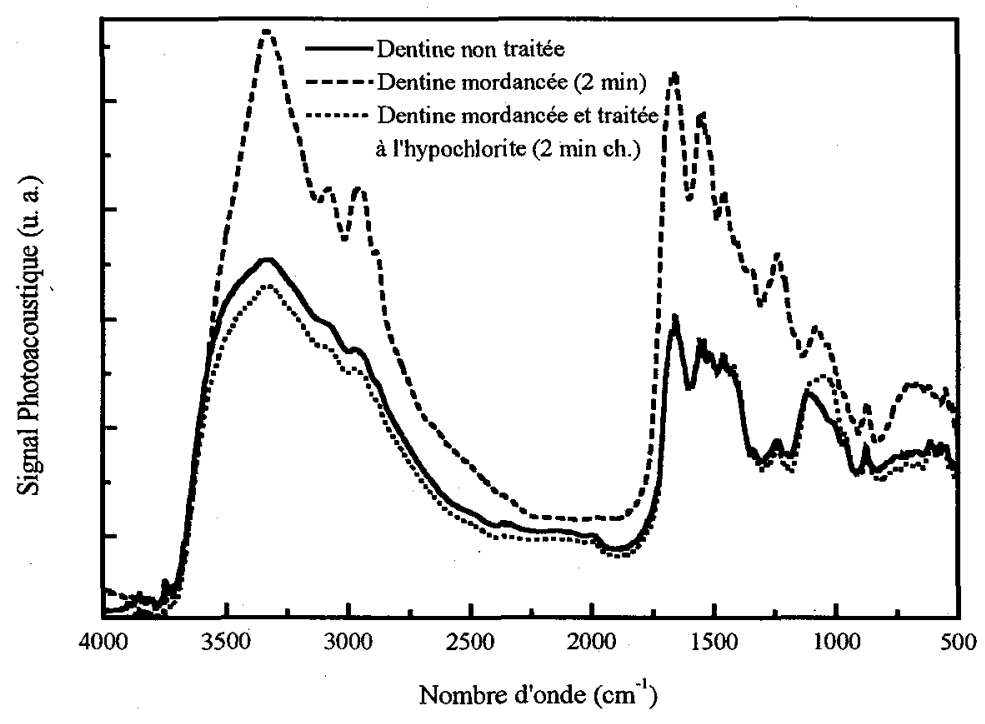

Figure 5: Spectres PA-FTIR du traitement de la dentine à l'acide et à l'hypochlorite de sodium.

Ces spectres indiquent l'enlèvement rapide de la phase organique préalablement exposée par l'acide. Après deux minutes de traitement à l'hypochlorite, le spectre est presque identique à celui de la dentine avant tout traitement. Les temps d'exposition plus longs (5 et 15 minutes) ne révèlent aucun autre changement.

\section{DISCUSSION}

Les spectres présentés à la figure 2 indiquent une augmentation de l'intensité du signal à travers toute la gamme spectrale. Cette croissance en fonction du temps de traitement est due à une augmentation importante du rapport surface/volume [24]. Au fur et à mesure que l'acide dissout l'HAP, des fibres de collagène sont exposées. Ceci résulte en une surface plus grande et donc un 
signal plus élevé. De plus, le taux de croissance devient moins important avec le temps. Ceci peut être le résultat d'un de deux effets ou d'une combinaison des deux. Le premier effet est un ralentissement de la réaction à cause de la diffusion nécessaire afin de déminéraliser plus profondément la surface de la dentine: la réaction de déminéralisation est contrôlée par diffusion, parce que l'interface minéralisée est de plus en plus loin de la surface initiale et donc le temps de diffusion est plus élevé, ce qui résulte en un temps de déminéralisation plus long. L'autre effet est la limite de la profondeur de détection de la technique photoacoustique: dans les conditions expérimentales de ce travail, la technique photoacoustique sonde à une profondeur approximative de quelques micromètres, et des études $[25,26,27]$ ont démontré que la profondeur de mordançage est aussi de quelques micromètres.

Tel que présenté précédemment, la perte de la phase minérale est claire. En se basant sur ces résultats ainsi qu'à d'autres travaux de ce groupe et d'autres $[10,11]$, le mordançage est plus rapide durant les deux premières minutes d'exposition aux acides. Le mordançage enlève l'hydroxyapatite de calcium ainsi que le carbonate apatite. De plus, l'acide citrique a un effet différent de l'acide maléique: son action est plus lente. Cet effet a déjà été remarqué dans notre étude par absorption atomique [10] et est dû à la formation de citrate de calcium à l'interface qui inhibe la réaction de décalcification. Puisque les spectres de l'acide phosphorique sont comparables à ceux de l'acide maléique, nous croyons que l'acide phosphorique crée des précipités mais ces derniers, en contraste à l'acide citrique, ne nuisent pas à la réaction de déminéralisation.

Le traitement de la dentine à l'hypochlorite implique un processus beaucoup plus lent. Les résultats indiquent non seulement la présence d'hydroxyapatite de calcium mais aussi la présence d'apatitie contenant du carbonate. Ces groupements carbonate peuvent remplacer soit les groupes hydroxyle ou phosphate du réseau cristallin. L'exposition de la dentine non mordancée à l'hypochlorite jusqu'à 15 minutes ne révèle presque aucun changement spectral. Les spectres de la dentine exposée pendant quelques jours indiquent encore la présence (quoique faible) de collagène. Il est clair que la perméation de l'hypochlorite à travers la dentine est très lente et plus hétérogène que le mordançage. Lorsque comparés aux résultats obtenus pour la dentine préalablement mordancée, il est clair que la déprotéination de la dentine est très rapide. Il est donc évident que l'enlèvement du collagène de la dentine est sévèrement inhibé par la présence du réseau cristallin de la phase minérale.

\section{CONCLUSIONS}

Le traitement de la dentine au moyen d'un acide décalcifie la surface très rapidement. La dissolution du collagène par l'entremise de l'hypochlorite de sodium est très lente. Cependant, lorsque la dentine est décalcifiée, le traitement à l'hypochlorite dissout rapidement le collagène exposé. La phase minérale de la dentine protège donc le collagène de l'attaque de l'hypochlorite de sodium. Ce travail démontre l'utilité de la spectroscopie PA-FTIR envers l'étude des surfaces et interfaces de tissus calcifiés. Cette technique s'avère un moyen puissant de déceler des changements chimiques advenant aux surfaces biologiques opaques et hétérogènes. 


\section{Remerciements}

Les auteurs désirent remercier le CRSNG du Canada pour leur financement et Mauro Di Renzo désire remercier le GCM pour une bourse d'étude.

\section{Références}

[1] Nakabayashi N., J. Jpn. Dent. Mater. 1 (1982) 78-86.

[2] Dickens-Venz S., Bowen R.L., Eichmiller F.C., J. Dent. Res. 72 (1992) Abst. No. 1201.

[3] Marshall G.W. Jr., Balooch M., Tench R.J., Kinney J.H., Marshall S.J., Dent. Mater. 9 (1993) 265-268.

[4] Stangel I., Sacher E., Young C., Hanley S., J. Adhesion 47 (1994) 133-149.

[5] Nakabayashi N., Internat. Dent. J. 35 (1985) 145-154.

[6] Di Renzo M., Ellis T.H., Domingue A., Bertrand L., Sacher E., Stangel I., J Adhesion 47 (1994) 115-121.

[7] Ruse N.D., Smith D.C., J. Dent. Res. 70 (1991) 1002-1008.

[8] Sterrett J.D., Delaney B., Rizkalla A., Hawkins C.H., Quintessence Int. 22 (1991) 371-375.

[9] Hennequin M., Pajot J., Avignant D., J. Endodon. 20 (1994) 551-554.

[10] Di Renzo M., Ellis T.H., Sacher E., Stangel I., J. Dent. Res. Soumis pour publication.

[11] Kinney J.H., Balooch M., Haupt Jr. D.L., Marshall S.J., Marshall Jr. G.W., J. Dent. Res. 74 (1995) 1179-1184.

[12] Spencer P., Byerley T.J., Eick J.D., Witt J.D., Dent. Mater. 8 (1992) 10-15.

[13] Stangel I., Ostro E., Domingue A., Sacher E., Bertrand L., "Photoacoustic Fourier transform IR spectroscopy of polymer-dentin interaction", Proceedings from the first international conference on polymer-solid interfaces. J.J. Pireaux, P. Bertrand, J.L. Bredas Eds. (Institute of Physics Publishing, Bristol, 1992), pp.157-167.

[14] Di Renzo M., Ellis T.H., Sacher E., Stangel I., Prog. Surf. Sci. 50 (1995) 407-418.

[15] Mixson J.M., Spencer P., Moore D.L., Chappel R.P., Adams S., Am. J. Dent. 8 (1995) 5-9.

[16] Fukuda K., Histochemie 6 (1966) 127-130.

[17] LeGeros R.Z., Trautz O.R., Klein E., LeGeros J.P., Experientia 25 (1969) 5-7.

[18] Young R.A., Clin. Orthop. Relat. Res. 113 (1975) 249-262.

[19] Montel G., Bonel G., Heughebaert J.C., Trombe J.C., Rey C., J. Crys. Growth 53 (1981) 74-99.

[20] Ishikawa K., Takagi S., Chow L.C., Ishikawa Y., Eanes E.D., Asaoka K., Dent. Mater. 10 (1994) 26-32.

[21] Stutman J.M., Posner A.S., Nature 193 (1962) 368-369.

[22] Winand L., Dallemagne M.J., Nature 193 (1962) 369-370.

[23] Blumenthal N.C., Posner A.S., Calc. Tiss. Res. 13 (1973) 235-243.

[24] Yang C.Q., Fately W.G., J. Mol. Struct. 141 (1986) 279-284.

[25] Erickson R.L., Am. J. Dent. 2 (1989) 117-123.

[26] Nakabayashi N., Takarada K., Dent. Mater. 8 (1992) 125-130.

[27] Stangel I., résultats non publiés. 